\title{
GW23-e2059 THE USE OF RETEPLASE IN PATIENTS WITH PULMONARY EMBOLISM RETEPLASE AFTER HAEMODYNAMIC CHANGES
}

doi:10.1136/heartjnl-2012-302920y.6

Zhaochuan Liu, Junling Wang. Department of Cardiology, No. 252 Hospital of PLA, Geriatric Cardiovascular Disease Center of Chinese PLA. Baoding, 071000, China

Objectives To explore the clinical effect of reteplase in the emergency use of thrombolytic therapy in patients with pulmonary embolism after haemodynamic changes.

Methods The patients with pulmonary embolism were confirmed by clinical symptoms and 320-slice spiral CT. After respiration, blood pressure and other haemodynamic changes, $20 \mathrm{mg}$ reteplase was used by a slow bolus two times with the intervals $30 \mathrm{~min}$ after the dilution. The haemodynamic changes and clinical symptoms were detected, while monitoring the hemagglutination application of heparin sodium into the continuous intravenous infusion. Results From December 2008 to January 2012, all patients with pulmonary embolism were confirmed by 320 row spiral CT in our hospital diagnosis of pulmonary embolism. 18 cases of the patients were selected to use reteplase to thrombolysis in the emergency application after haemodynamic changes appeared in them. At $6 \mathrm{~h}$ after thrombolysis, chest pain and dyspnoea was significantly improved in 12 cases (66\%); blood pressure, heart rate, breathing restored normal within $24 \mathrm{~h}$ in 13 cases (72\%); At $2 \mathrm{~h}$ after thrombolysis, one patients died in cerebral haemorrhage (5.5\%); at $6 \mathrm{~h}$ after thrombolysis, four patients whose haemodynamics was not improved, died eventually (22\%). At 1 week after thrombolysis, pulmonary three-dimensional imaging examinations showed recanalisation in 13 cases (72\%) of patients with stable disease underwent. The rates of recanalisation and mortality in patients with pulmonary embolism were statistically significant $(p<0.05)$. 


\section{ABSTRACTS}

Conclusions When the patients with deep vein thrombosis, lower extremity fracture, surgery, prolonged bed rest, old age, pregnancy and childbirth, obesity, hypertension and other underlying diseases, appeared unexplained dyspnoea, chest pain, syncope, transient reduced blood pressure, oxygen partial pressure gradually lower, pulmonary embolism should be suspected. The patients are in critical condition and can lead to death with the rapid development in a short period of time due to circulatory, respiratory system failure. So using the emergency thrombolysis as soon as to pulmonary vascular recanalisation is an effective treatment programme. The patients with pulmonary embolism and haemodynamic changes confirmed in our hospital are given with reteplase to thrombolysis. The drug has the clinical safety, and can significantly relieve symptoms, improve haemodynamic changes and increase pulmonary vascular recanalisation rate. It improves the cure rate of patients and reduce mortality effectively. 